\title{
Visual object-oriented technology and case-tools of developing the Internet / Intranet-oriented training courses
}

\author{
Salaimeh S. A. , Hjouj A. A. \\ Aqaba University of Technology, 79 Wasti al-Tal St., 11191, Amman, Jordan
}

\author{
Article info: \\ Paper received: \\ The final version of the paper received: \\ Paper accepted online:
}

\author{
November 20, 2017 \\ December 1, 2017 \\ December 3, 2017
}

*Corresponding Author's Address: safwan670@yahoo.com

\begin{abstract}
New information technologies, modern computers, LAN, WAN networks enable us to modernize the whole education system. One of the most perspective ways of the modern educational system's development is online education. The questions of developing the visual instrumental system PIECE designed to automate processes of creation the cross- platform hypermedia training and controlling course (HTCC) are viewed in this paper.
\end{abstract}

Keywords: platform, training course, hypermedia, tools, educational system, modernization.

\section{Introduction}

New information technologies, modern computers, LAN, WAN networks enable us to modernize the whole education system. One of the most perspective ways of the modern educational system's development is online education.

Many major international corporations, such as oracle, Microsoft, etc. have their training centers all over the world. They enable to provide online education via internet and e-mail. In the most of highly developed countries the great deal if instruction by correspondence is based on the online training.

In Jordan online education is in the first stages. Now the main obstacles are the lack of necessary laws and highly developed state infrastructure.

In order to subdue the difficulties of crisis in modern education service, now the most perspective trends of scientific exploration and practical works is to create methods, tools and information technologies, providing effective and high grade online education, electronic training information interchange, interactive computer based both control and self- control of student's knowledge [1,2].

\section{Methods}

\subsection{The tools of online education}

Online education is design to enhance to the efficiency of providing the education services, using the modern computer equipment telecommunication systems. New information technologies and tools of its providing.

There are some base classes of the modern tools used for enhance the efficacy of online education, such as:

- electronic publications;

- online practical and laboratory works;

- computer - based training systems with remote access;

- online database and knowledge - base;

- electronic libraries with remote access;

- audio and video information training stuff;

- geo information teaching systems;

- tools for creation and filling them with training information.

\subsection{Technology of development training system PIECE}

The questions of developing the visual instrumental system PIECE designed to automate processes of creation the cross- platform hypermedia training and controlling course (HTCC) are viewed in this paper. The main principles of realizing HTCC are these requirements: 
- system has to be cross - platform, i.e. common orientation to using created HTCC at any type of computer and in any operating system, which has embedded tools of Java virtual machine (JVM) support;

- hypertext and multimedia support;

- it has be easy to create and add new training courses, to replace and delete outdated fragments;

- orientation for no programmers users, e.g. tutors of humanitarian;

- media, technical subjects, etc.;

- executable code of active training components (e.g. subsystem of control and self - control) has to be executed while shipped to the end - user (student);

- high-level security.

HTCC are the hypertext and multimedia HTML - files (declarative component) with the embedded Java -apples (executable active component), oriented to usage at the various apparatus and program platforms in Internet/Internet networks, and also in the file-server LAN or (if necessary) at a single computer [3-5].

The modern object-oriented Java language, Providing developing of the cross-platform software, and HTML, to create bright and colorful hypertext and multimedia training courses, practical works and controlling environments, altogether as a set of interrelated Web-pages were chooser as the base tools of realizing the suggesting method. Relations between components can be set both in syntax and semantic-logical levels, matches the proper professional knowledge about studied subject.

\subsection{Functional Structure of Piece 1.0}

Base functional component is an interactive intellectual instrumental environment, designed to create new hypermedia training courses. It contains set of tools and adjustable system of wizards. They help to manage the system during automatic generation of source code mode of training courses. Also they order, regulate and make easy processes of filling out databases and knowledge bases of created HTCC with structured training information of specific data domain. The powerful and adaptable environment of visual programming in Java language Symantec Visual Café Database Development Edition 3.0 was used to realize this component.

System-shell PIECE consist of 3 base functional components (Fig.1).

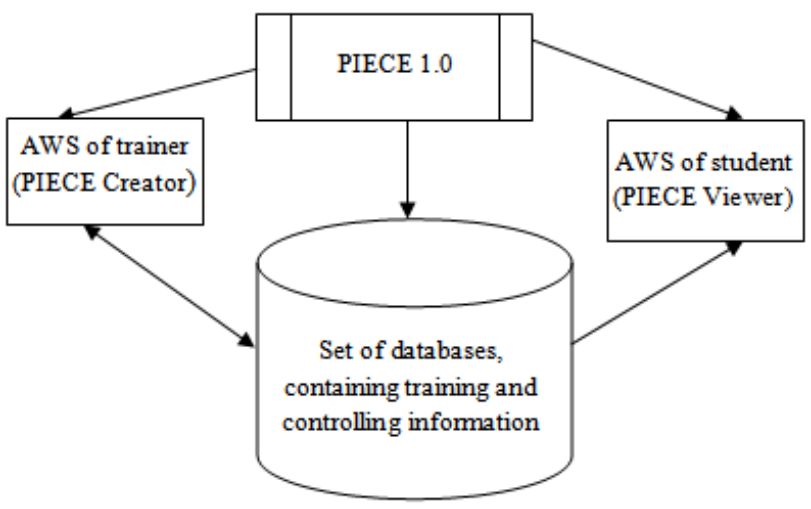

Figure 1 - Schema of functional structure of PIECE
Base component provides solving of the two main functional problems of HTCC synthesis:

- how to create and edit adaptable tests, including various types of answers;

- how to create, add and delete structured hypermedia information in training courses.

When solving the first problem, trainer can create convenient additional component of automated control and self-control of students' knowledge, possible online. PIECE supports 4 main types of questions. They are:

- choosing one right answer from many others;

- choosing some right answers from many others;

- string input;

- number in some range input.

In future number of types of the questions will grow, according to the requirements of corresponding training data domain. The environment has intuitive interface, contains wizards, which direct actions of developer and enables users with insufficient preliminary (the system is designed for users with various preliminary level) easily and quickly develop their own controlling tests.

Solution to the second problem of synthesis HTCC enables users to create structured information contents of course as a system of interrelated training Web-pages with embedded Java-applets, and also files, contains test questions and variants of its answers. There are also instructions on designing the courses. After successful solution to the problems of functional synthesis HTCC of specific data domain, PIECE automatically extends its interface, adding new menu item. Also PIECE automatically create new folder and copy all files in it, related to the new training course. Andin conclusion, PIECE sends parameters of the knowledge verify to the controlling program.

Second functional component is a set of special ordered files and its service programs. It is called repository (system database). Repository contains information about all new changes designed by PIECE courses or about courses already in use. Data about each course registered in the system are situated in a single folder, inside the main folder PIECE. All the relations between files are set inside this folder, that's why after creating new HTCC it is enough to move new folder to the appropriate Webserver, local file-server or to the single computers, used for training.

Third component is a final product i.e. specific instance of HTCC, created during dialog with trainer via base component of PIECE. Generated HTCC is a filled with a training information set of hypermedia files, includes embedded cross-platform executable code in the form of Java-applets, intended to be executed while shipped to the students computer. HTCC is oriented to the comfortable and ergonomic presentation of the training information and as well as to the organization of effective automated control and self-control of students' knowledge. After reviewing each fragment of training hypertext and work with active and passive multimedia 
information and reference subsystems user can go on to the verification of obtained knowledge. User will pass the set of tests in this mode. And according to the answers he will get integrated mark.

Developing environment (base component of PIECE) can be executed from Visual Cafe or from the applet viewer of Sun Java Development of Sun Java Development Kit (JDK) 1.1.x. After interactive synthesis of the second component it has to be moved to the server, where programs of knowledge verification it has to be moved to the server, where programs of knowledge verification and also set of web-pages, which forms the main homepage of PIECE are situated [6-9].

When handling the web-site through Internet, where PIECE is located, user remotely reads training hypertext, supplied (if necessary) with multimedia fragments. Also he can test his knowledge. Program will be executed while shipped to the client's computer. In Intranet networks this process is the same. In file-server LANs, working under such operating systems, as Novell Netware3.x or 4.x, base component of PIECE has to be located at the file-server. Then it can be opened as an ordinary Web-page with the help of browser with Java supply. It is possible to use PIECE at a single local computer. This time it is necessary to open Web-page located at the user's hard disk in browser.

\subsection{The basic technological phases of working with PIECE 1.0}

First technological phase is creating test questions file on selected topics. Menu item File/Create, located at the first dialog window of the wizard, is used to pass this phase. While forming the test question user has to define its type. Next dialog Window has to contain the text of the question and the proper image for it. To view previously created questions select menu item Question/View all to save file of test questions select menu item File/Save. The extension must be *.quest. This wizard is also used to create answers on questions.
Menu item Link the whole training course is used for linking altogether the components of the training course. To add an icon of new course into main menu of PIECE it is necessary to select Course/Add menu item and set a unique identifier of the course. After that a anew folder will appear inside the PIECE folder. The main training file, file of questions, demo examples and generated Web-pages, serving the course will be copied there. To install PIECE into the Web-server it is enough to copy folder containing PIECE. Thus training course can be created locally and published in the Web-server.

Student has to open index.html file in the root directory of PIECE folder to read all the courses, included into the PIECE. It is Possible to do so on the Web-site, as well as at the LAN at a single machine.

\section{Conclusions}

The closest perspective for system enhancement is the development of a subsystem. Its Purpose is to manage the processes of automated filling the created courses with training information, that is based on calling the system of interrelated dynamic templates and responding wizards set, adaptively. They are needed for supporting processes of interactive input of training information and for creating the screen forms with embedded hypermedia inserts. At the moment trainer-developer using this subsystem can either choose the proper wizard, or apply adaptive and simple script language to realize tools of the training information reflection. Actual questions are also the realize tools of the training information reflection. Actual questions are also the setting of the security system, running the individual or class electronic log.

The questions of developing the visual instrumental system PIECE designed to automate processes of creation the cross- platform hypermedia training and controlling course (HTCC) are viewed in this paper.

\section{References}

1. Salaimeh, S. A., Batiha, Kh. (2006). Business Process Simulation with Algebra Event Regular Expression. Information Technology Journal, Vol. 5, No. 3, 583-589.

2. Batiha, Kh., Salaimeh, S. A. (2006). E-Learning. Leonardo Electronic Journal of Practices and Technology, pp. 1-4.

3. Batiha Kh., Salaimeh, S. A., Besoul, Kh. A. (2006). Digital Art and Design. Leonardo Journal of Science, pp 1-8.

4. Salaimeh, S. A., Zaher, A. A. (2011). Developing enterprise system with CORBA and JAVA integrated technologies. Journal annals computer science series, Vol. 9, No. 1.

5. Salaimeh, S. A., Zaher, A. A. (2011). Using Java Technologies in Developing Enterprise Systems. Australian Journal of Basic and Applied Sciences.

6. Salaimeh, S. A., Saraireh, Z. A., Rawashdeh, J. H. A. (2015). Design a Model of Language Identification Tool. International Journal of Information \& Computation Technology, Vol. 5, No. 1, 11-18.

7. Michael, J. L. (2017). Object-Oriented Programming Featuring Graphical Applications in Java.

8. Sarcar, V. (2016). Interactive Object Oriented Programming in Java: Learn and Test Your Skills, Apress.

9. Sanders, K. E., Bam, A. V. (2005). Object-oriented programming in Java a graphical approach, Addison Wesley. 